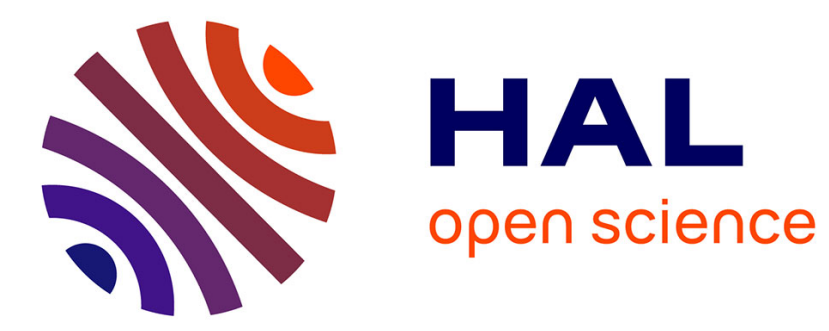

\title{
Élargissement par les dislocations des pseudo-lignes de Kikuchi
}

\author{
P. Guyot
}

\section{To cite this version:}

P. Guyot. Élargissement par les dislocations des pseudo-lignes de Kikuchi. Revue de Physique Appliquée, 1974, 9 (2), pp.393-397. 10.1051/rphysap:0197400902039300 . jpa-00243793

\section{HAL Id: jpa-00243793 https://hal.science/jpa-00243793}

Submitted on 1 Jan 1974

HAL is a multi-disciplinary open access archive for the deposit and dissemination of scientific research documents, whether they are published or not. The documents may come from teaching and research institutions in France or abroad, or from public or private research centers.
L'archive ouverte pluridisciplinaire HAL, est destinée au dépôt et à la diffusion de documents scientifiques de niveau recherche, publiés ou non, émanant des établissements d'enseignement et de recherche français ou étrangers, des laboratoires publics ou privés. 


\title{
ÉLARGISSEMENT PAR LES DISLOCATIONS DES PSEUDO-LIGNES DE KIKUCHI (*)
}

\author{
P. GUYOT \\ Laboratoire de Thermodynamique et Physico-chimie Métallurgiques \\ LA29, ENSEEG, B. P. 44, 38401 Saint-Martin-d'Hères, France
}

\begin{abstract}
Résumé. - Les distorsions de réseau cristallin introduites par les dislocations diminuent le contraste et augmentent la largeur des pseudo-lignes de Kikuchi observées au microscope électronique à balayage. Un modèle est présenté reliant la largeur des lignes à la densité des dislocations sous-jacentes à la surface de l'échantillon, en accord satisfaisant avec les résultats expérimentaux obtenus pour l'aluminium.
\end{abstract}

\begin{abstract}
The lattice distorsions induced by the dislocations decrease the contrast and widen the channelling lines observed in a scanning electron microscope. A model is proposed relating the line width to the dislocation density lying under the specimen surface, in satisfying agreement with the experimental results obtained for aluminium.
\end{abstract}

1. Introduction. - Les dislocations ont récemment été résolues au microscope électronique à balayage en transmission sur lames minces, à une tension d'accélération de $80 \mathrm{kV}$, en émission thermo-électronique d'un filament de tungstène [1], [2], et à $50 \mathrm{kV}$ avec un filament d'hexaborure de lanthane [3]. En réflexion, les images sont de mauvaise qualité, ou inexistantes, par suite des diffusions multiples, sur échantillon massif.

Les images des dislocations ont été calculées en théorie dynamique par Clarke et Howie [4], leur permettant ainsi de conclure qu'en mode rétrodiffusé, les microscopes conventionnels (cathode chaude, tension d'accélération maximum de $30 \mathrm{kV}$, détecteur conventionnel) sont incapables de résoudre les dislocations sur échantillon massif dans des durées de balayages raisonnables. Ceci se prévoit facilement si l'on considère que le profil d'intensité d'une dislocation coin de vecteur de Burgers parallèle à la surface de l'échantillon doit être identique à celui d'une pseudo-ligne de Kikuchi. En effet, une pseudoligne de Kikuchi est excitée dans le cristal parfait quand le faisceau d'électrons incident oscille de part et d'autre de l'angle de Bragg $\theta_{\mathbf{B}}$ des plans cristallins considérés, figure $1 a$. La situation est la même pour un faisceau parallèle arrivant sous incidence de Bragg sur le cristal contenant la dislocation, figure $1 b$; l'écart d'interférence est dans le premier cas $s_{g}=g\left(\theta-\theta_{\mathrm{B}}\right)$, dans le second $s_{g}=g \theta_{\perp}$, où $\mathbf{g}$ et $\theta_{\perp}$ sont respectivement le vecteur de réflexion et la rotation des plans cristallins introduits par la dislocation. Par conséquent, le profil d'intensité d'une pseudoligne de Kikuchi, représenté sur la figure $1 c$ sera aussi celui de la dislocation. Minimum et maximum

$\left(^{*}\right)$ Broadening of channelling pattern lines by dislocations.

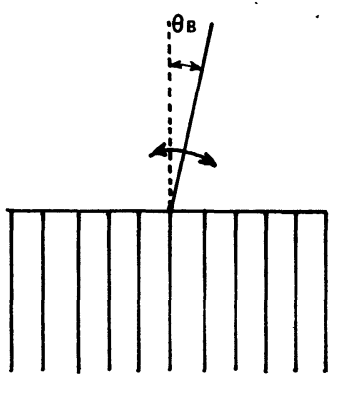

a

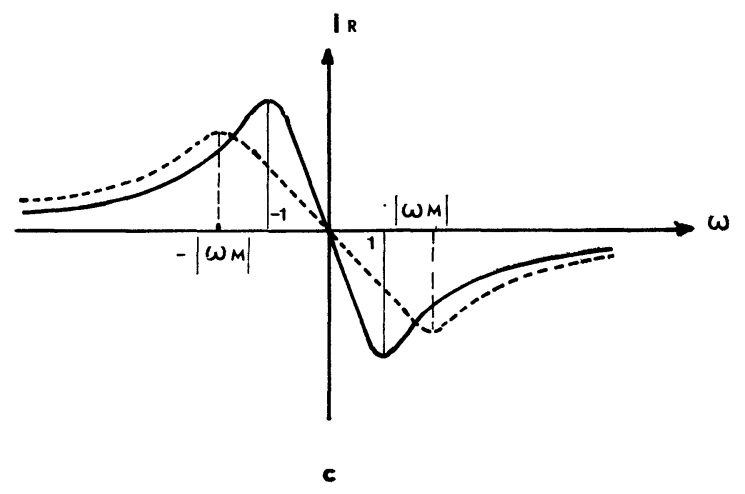

FIG. 1. - $-a$ ) et $b$ ) Analogie entre pseudo-ligne de Kikuchi et dislocation (cf. texte). $c$ ) Profil d'orientation $I_{\mathrm{R}}$ en fonction de $\omega=S_{g} \xi_{g}$. Trait plein : faisceau incident parallèle. Trait pointillé : faisceau incident divergent.

d'intensité se produidant pour $\omega=s_{g} \xi_{g}= \pm 1$ ( $\xi_{g}=$ distance d'extinction de la réflexion considérée), on en déduit la largeur $\Delta x$ de l'image de la dislocation, compte tenu que $\theta_{\perp} \sim 7 b / 16 \pi x(x=$ distance au cœur de la dislocation, parallèlement à la surface) :

$$
\frac{1}{g \xi_{g}}=\frac{7 b}{16 \pi(\Delta x / 2)}
$$


soit

$$
\Delta x \sim 0,3 \quad g b \xi_{g} .
$$

D'où la double condition imposée au faisceau incident pour la résolution de la dislocation :

- diamètre $D<\Delta x$,

- divergence $2 \alpha<$ largeur angulaire de l'image $=2 / g \xi_{g}$.

Avec une brillance $B$ de cathode chaude de $4 \times 10^{4} \mathrm{~A} \mathrm{~cm}^{-2} \mathrm{sr}^{-1}$, on déduit une intensité disponible

$$
\begin{aligned}
I & \sim 2,5 B D^{2} \alpha^{2} \\
& \sim 0,225 . B b^{2} \\
& \sim 0,9 \times 10^{-11} \mathrm{~A}
\end{aligned}
$$

c'est-à-dire insuffisant pour détecter en $20 \mathrm{~s}$ un contraste de $5 \%\left(\sim 10^{-9} \mathrm{~A}\right)$.

L'émission de champ $\left(B \sim 10^{8} \mathrm{~A} \mathrm{~cm}^{-2} \mathrm{sr}^{-1}\right)$ est donc nécessaire à l'observation des dislocations au voisinage de la surface d'un échantillon massif.

La résolution des dislocations étant impossible avec un microscope à balayage conventionnel, on est limité dans la caractérisation de l'état de déformation superficielle, à intégrer les distorsions du cristal perçues par le faisceau incident, qui ont pour effet d'élargir les résonances de Bragg, c'est-à-dire les pseudo-lignes de Kikuchi [5].

Nous proposons ici un modèle reliant la largeur des lignes à la densité de dislocations responsables des distorsions.

2. Elargissement des pseudo-lignes de Kikuchi. Le profil d'orientation en réflexion, c'est-à-dire la variation de l'intensité réfléchie relative $I_{\mathrm{R}}$ en fonction de l'angle d'incidence $\theta$ (ou de $\omega$ ) est représenté, pour un faisceau incident parallèle et un cristal parfait, sur la figure 1c. Il correspond à l'équation, calculée à partir d'une théorie dynamique à deux faisceaux [6] :

$$
I_{\mathrm{R}}(\omega)=1-\frac{\omega}{\left(1+\omega^{2}\right)}
$$

d'où la largeur angulaire de la ligne, couvrant la variation d'incidence entre maximum $(\omega=-1)$ et minimum $(\omega=+1)$ de $I_{\mathrm{R}}$ :

$$
2 W_{0}=2 / g \xi_{g} \text {. }
$$

Le profil d'orientation pour un faisceau incident de divergence $2 \alpha$ s'obtient en moyennant, pour chaque incidence $\theta$ du faisceau, $I_{\mathrm{R}}$ entre $\theta-\alpha$ et $\theta+\alpha$. La conséquence est que la largeur de la ligne augmente et que son contraste diminue ([6], Fig. 1).

Considérons maintenant un cristal contenant une densité $\rho$ de dislocations coins parallèles à la surface. Un faisceau parallèle de diamètre $\rho^{-1 / 2}$ voit une dislocation unique, figure $1 b$; les rotations des plans cristallins de part et d'autre de la dislocation jouent ici le même rôle que la divergence du faisceau précé- demment considéré. Il en résulte un élargissement de la pseudo-ligne de Kikuchi qu'il est facile d'évaluer.

L'intensité rétrodiffusée moyenne s'écrit, pour une incidence particulière du faisceau incident :

$$
<I_{\mathrm{R}}(\omega)>=\frac{1}{2 \Delta \omega} \int_{\omega-\Delta \omega}^{\omega+\Delta \omega} I_{\mathrm{R}}(\omega) \mathrm{d} \omega
$$

avec

$$
\Delta \omega=\xi_{g} g<\theta_{\perp}>
$$

$\left\langle\theta_{\perp}\right\rangle$ représente, de part et d'autre de la dislocation, la rotation moyenne des plans cristallins réflecteurs dans un cristal de largeur $\left(\rho^{-1 / 2} / 2\right)$.

La largeur $2 W$ de la pseudo-ligne de Kikuchi est déterminée par les points $\omega_{\mathbf{M}}$ pour lesquels

$$
\frac{\mathrm{d}<I_{\mathrm{R}}(\omega)>}{\mathrm{d} \omega}=0
$$

soit compte tenu de (1) et (2) :

$$
\omega_{M}= \pm\left(1+\Delta \omega^{2}\right)^{1 / 2}
$$

qui conduit à :

$$
2 W=\frac{2}{g \xi_{g}}\left|\omega_{M}\right|=\frac{2}{g \xi_{g}}\left(1+g^{2} \xi_{g}^{2}<\theta_{\perp}>^{2}\right)^{1 / 2}
$$

soit :

$$
2 W=2\left(W_{0}^{2}+<\theta_{\perp}>^{2}\right)^{1 / 2} .
$$

La dislocation coin de la figure $1 b$ étant suivant l'axe $O_{y}, O_{z}$ étant vertical, la rotation des plans cristallins est :

$$
\begin{aligned}
& \theta_{\perp}(x, z) \sim \frac{\partial R_{x}}{\partial z}= \\
& \quad=\frac{b}{2 \pi} \frac{x}{x^{2}+z^{2}}\left(1+\frac{1}{2(1-v)} \frac{x^{2}-z^{2}}{x^{2}+z^{2}}\right)
\end{aligned}
$$

$\boldsymbol{R}$ étant le vecteur déplacement de la dislocation. Le volume $V$ total de cristal associé à l'unité de longueur de dislocation étant $\left(\rho^{-1}\right)$ l'on a :

$$
<\theta_{\perp}>2 \rho \iint_{V / 2} \theta_{\perp}(x, z) \mathrm{d} x \mathrm{~d} z
$$

Soit après calcul

$$
<\theta_{\perp}>\sim \frac{b}{2 \pi} \rho^{1 / 2}\left(\frac{\pi}{4}+\frac{7}{8} \log 2\right) \sim 0,22 b \rho^{1 / 2} .
$$

Le faisceau incident ayant en réalité un diamètre $D$ supérieur à $\rho^{-1 / 2}$ il est nécessaire de calculer la désorientation $\Theta$ introduite par les dislocations d'un bord à l'autre de la région explorée. La résonance de Bragg d'une ligne étant de l'ordre de $10^{-3} \mathrm{rd}$, les aberrations sphériques sont négligeables, si bien que la région explorée correspondante a un diamètre sensiblement égal à $D$. 
$\perp \int_{\frac{1}{1} p^{-1 / 2}}^{\perp} \perp \perp \perp$

a

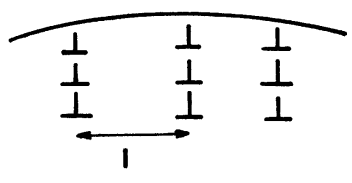

d $\perp T \perp T \perp \perp$ la surface.

Les électrons rétrodiffusés participant à la formation des lignes provenant d'une profondeur sous la surface du cristal d'environ $500 \AA$, c'est-à-dire inférieure à la distance moyenne $\rho^{-1 / 2}$ entre dislocations pour les densités expérimentalement détectées (voir $\S 3$ ), on pourra négliger en première approximation les dislocations situées à plus de $\rho^{-1 / 2}$ de la surface. La configuration $a$ ) est représentée sur la figure $2 a$.

Dans ces conditions, les désorientations s'ajoutent d'un bord à l'autre du faisceau :

$$
\left.\dot{\Theta}=\left(\frac{D}{\rho^{-1 / 2}}\right)<\theta_{\perp}\right\rangle \text {. }
$$

Nous considérons pour évaluer $\Theta$ quatre configurations de dislocations coins :

a) Dislocations de même vecteur de Burgers et

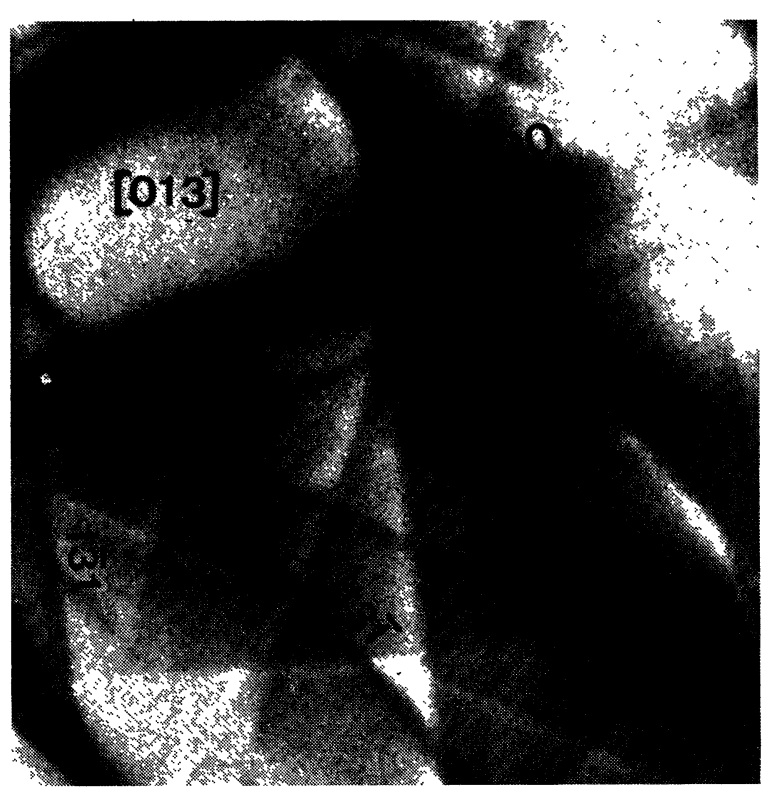

(a)

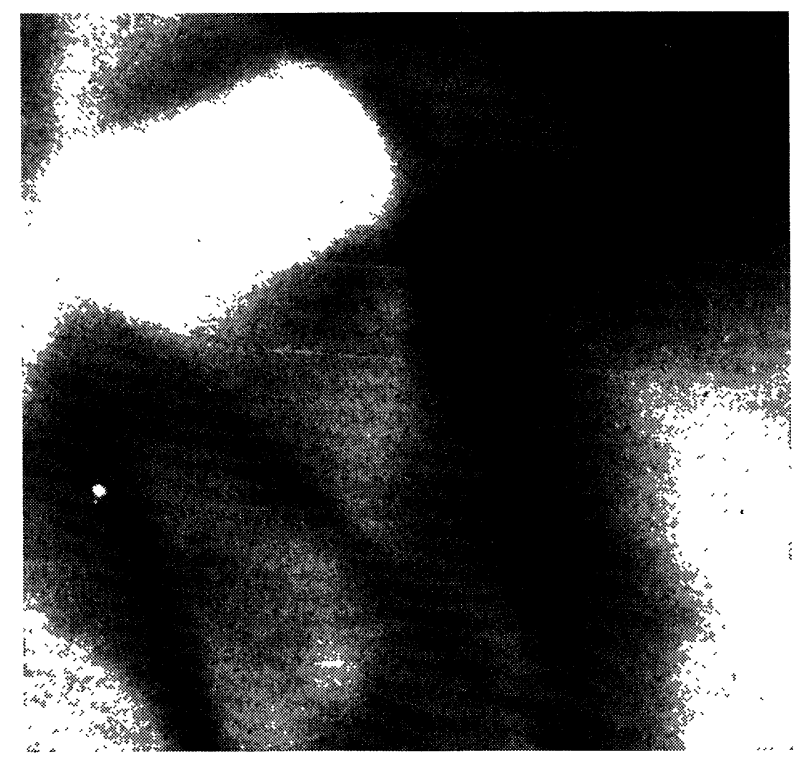

Planche 1. - Elargissement des lignes en fonction du taux de déformation plastique $\varepsilon . a) \varepsilon=0 \% ; b) \varepsilon=4 \% ; c) \varepsilon=6 \%$. Condition de travail du microscope Cambridge $\mathrm{S} 4$ : diamètre du faisceau sur l'échantillon : 1 à $2 \mu \mathrm{m}$; tension d'accélération $30 \mathrm{kV}$; mode micro-Kikuchi. 
Il y a alors compensation partielle des désorientations [7] :

$$
\Theta=\left(\frac{D}{\rho^{-1 / 2}}\right)^{1 / 2}<\theta_{\perp}>
$$

c) Dislocations réparties en sous-joints perpendiculaires à la surface et de même signe (Fig. 2c). Si $l$ est la taille des sous-grains :

$$
\Theta=\left(\frac{D}{l}\right) \rho l b=D \rho b
$$

d) Même configuration qu'en $b$ ), mais avec des sous-joints de signe variable (Fig. $2 d$ ) :

$$
\Theta=\left(\frac{D}{l}\right)^{1 / 2} \rho l b=D^{1 / 2} l^{1 / 2} \rho b .
$$

On obtient la largeur des lignes en remplaçant dans l'expression (4) $\left\langle\theta_{\perp}\right\rangle$ par les différentes expressions de $\Theta$, soit :

$$
\begin{aligned}
& 2 W=2\left[\left(g \xi_{g}\right)^{-2}+0,05 D^{2} \rho^{2} b^{2}\right]^{1 / 2} \\
& 2 W=2\left[\left(g \xi_{g}\right)^{-2}+0,05 D \rho^{3 / 2} b^{2}\right]^{1 / 2} \\
& 2 W=2\left[\left(g \xi_{g}\right)^{-2}+D^{2} \rho^{2} b^{2}\right]^{1 / 2} \\
& 2 W=2\left[\left(g \xi_{g}\right)^{-2}+D l \rho^{2} b^{2}\right]^{1 / 2} .
\end{aligned}
$$

3. Résultats expérimentaux et discussion. - L'élargissement des lignes a été mesuré sur des polycristaux d'aluminium 99,95\% (taille des grains $\sim 100 \mu \mathrm{m}$ ) déformé en traction.

Les lignes ont été formées par les électrons rétrodiffusés, en mode "micro-Kikuchi » (point d'oscillation du faisceau sur la surface de l'échantillon).

La planche 1 illustre l'élargissement des lignes observé en fonction du taux de déformation plastique $\varepsilon$. L'évaluation de la densité $\rho$ des dislocations par microscopie électronique en transmission permet de tracer les variations de la largeur des lignes en fonction de $\rho$. Les variations de la largeur de ligne (131) sont ainsi représentées sur la figure 3 ; les courbes correspondent aux expressions théoriques (7a) et $(7 b)$ pour les valeurs de $D$ indiquées.

Compte tenu des conditions opératoires présentement utilisées $(D \sim 1$ à $2 \mu \mathrm{m})$, on constate pour $\varepsilon<8 \%$ que l'expression (7a) est en bon accord avec les résultats expérimentaux. La configuration $b$ ), correspondant aux dislocations de signes alternés, donc moins probable, conduit à des largeurs trop faibles.

Pour $\varepsilon>8 \%$ les dislocations s'organisent en cellules [8]; pour $\varepsilon=10 \%$ la taille des cellules est voisine $\mathrm{du} \mu \mathrm{m}$; la désorientation entre sous-grains étant de l'ordre de $0,8^{\circ}$, on calcule d'après $(7 b)$ ou

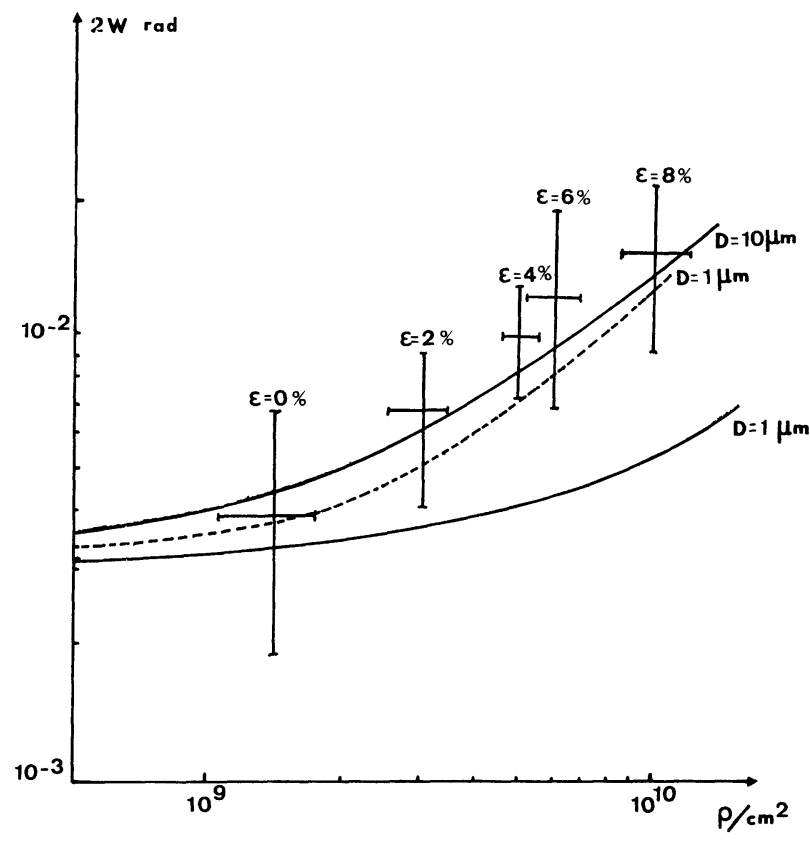

FIG. 3. - Variations de la largeur de la ligne (131) en fonction de $\rho$ dans Al. Les courbes en trait plein correspondent à l'expression $(7 b)$, en trait pointillé à $(7 a)$.

(7c) une largeur de ligne (131) de $2,8 \times 10^{-2} \mathrm{rd}$, valeur en accord satisfaisant avec la valeur expérimentale de $1,9 \times 10^{-2}$ rd.

Notre modèle, bien que basé sur des configurations de dislocations relativement simplistes, semble expliquer correctement les résultats expérimentaux obtenus avec l'aluminium. Il serait évidemment intéressant de réaliser des structures d'écrouissage plus pures que celles présentement obtenues sur un polycristal. Il y aurait également lieu d'introduire dans le modèle des dislocations vis, et de tenir compte des effets de relaxation de la surface.

Pour conclure, nous voyons que la méthode est sensible à des densités de dislocations relativement importantes et de l'ordre de $10^{9} \mathrm{~cm}^{-2}$. Il y a intérêt, pour améliorer la sensibilité de la méthode, à travailler avec des lignes de hauts indices, dont la largeur théorique, $2\left(g \xi_{g}\right)^{-1}$, est petite. Enfin les faibles densités de dislocations doivent être accessibles en utilisant les lignes de Kossel, dont l'élargissement par déformation a été observé qualitativement par Dingley [9] (bien que les temps de pose importants ainsi que la plus grande dimension de la région explorée constituent dans ce cas des inconvénients qui n'existent pas pour les pseudo-lignes de Kikuchi).

Remerciements. - Nous remercions M. J. M. Gjurasevic (Centre de Recherches Pechiney, Voreppe), pour son assistance technique dans la réalisation expérimentale du présent travail. 


\section{Bibliographie}

[1] Clarke, R. D., Phil. Mag. 24 (1971) 973.

[2] Humphreys, C. J., SPencer, J. P., Woolf, R. J., Joy, D. C., TitchMarsh, J. M., Booker, G. R. : SEM Proceedings of the fifth annual Scanning Electron Microscope Symposium, Chicago (1972).

[3] Stern, R., Revue Phys. Appl. 9 (1974) 377.

[4] Clarke, D. R., Howie, A., Phil. Mag. 24 (1971) 959.

[5] Davidson, S. M., Booker, G. R., Stickler, R., Proc. 25th Anniversary Meeting of EMAG, Inst. Physics, 298 (1971).

[6] Schulson, E. M., Phys. Stat. Sol. 46 (1971) 95.

[7] Friedel, J., Dislocations (Pergamon Press) 1964, p. 416.

[8] Guyot, P., GuURasevic, J. M., Journal de Microscopie (sous presse).

[9] Dingley, D. J., Proc. 25th Anniversary Meeting of EMAG, Inst. Physics, 206 (1971). 